\title{
Population-level risk factors for vertical transmission of HIV in the national prevention of mother-to-child transmission programme in South Africa: An ecological analysis
}

\author{
F Moyo, ${ }^{1,2,3}$ MSc, PhD; A Haeri Mazanderani, ${ }^{1}$ MB BCh, PhD; G G Sherman, ${ }^{1,3,4}$ MB BCh, PhD; T Kufa, ${ }^{1,2}$ MB BCh, PhD \\ ${ }^{1}$ Centre for HIV and STIs, National Institute for Communicable Diseases, National Health Laboratory Service, Johannesburg, South Africa \\ ${ }^{2}$ School of Public Health, Faculty of Health Sciences, University of the Witwatersrand, Johannesburg, South Africa \\ ${ }^{3}$ Paediatric HIV Diagnostics, Wits Health Consortium, Johannesburg, South Africa \\ ${ }^{4}$ Department of Molecular Medicine and Haematology, Faculty of Health Sciences, University of the Witwatersrand and National Health \\ Laboratory Service, Johannesburg, South Africa
}

Corresponding author: F Moyo (faith.moyo@yahoo.co.za)

\begin{abstract}
Background. Although South Africa has an overall mother-to-child transmission (MTCT) of HIV rate $<5 \%$, case rates remain high. Objectives. To identify population-level predictors of MTCT to inform targeted interventions to further reduce paediatric HIV incidence. Methods. The study was an ecological analysis of routine laboratory HIV-related test data from a synthetic cohort of women of reproductive age living with HIV (WRLHIV), identified from the National Health Laboratory Service's Corporate Data Warehouse between 2016 and 2017. Criteria based on syphilis screening and timing of HIV-related tests were used to identify pregnant and non-pregnant WRLHIV. Pregnant WRLHIV were followed from cohort entry at the first antenatal care (fANC) visit, through delivery to exit at the latest viral load (VL) or 15 months post delivery. Follow-up for non-pregnant WRLHIV started at cohort entry on 1 January 2016 to exit at the latest VL or 31 December 2018. HIV VL tests performed at cohort entry, delivery and cohort exit described viraemia (VL $\geq 50$ copies $/ \mathrm{mL}$ ) at subdistrict level. A negative binomial regression model determined the association between MTCT cases and the number of viraemic WRLHIV at different time points, controlling for number of WRLHIV aged $<25$ years at cohort entry and other routine HIV-related indicators at subdistrict level.

Results. Of 3386507 WRLHIV identified, 178319 (5.3\%) met criteria for pregnancy. Median (interquartile range (IQR)) proportions of women with fANC booking <20 weeks' gestation, maternal HIV seroprevalence during antenatal care (ANC) and antiretroviral therapy (ART) coverage during ANC were 68.2\% (62.9 - 72.8), 31.5\% (23.4 - 35.7) and 94.8\% (89.7 - 97.8), respectively. Viraemia was consistently higher in pregnant v. non-pregnant WRLHIV at median proportions of $42.9 \%(38.3-59.3)$ v. $35.0 \%(25.9-49.0)$ at cohort entry $(p<0.001)$ and $36.3 \%(25.0-48.4)$ v. $29.6 \%(21.0$ - 42.6) at cohort exit $(p<0.001)$. In total, 4535 children aged $<24$ months tested HIV polymerase chain reaction-positive, representing a median subdistrict-level case rate of 1372 (914 - 2 077) per 100000 live births. Maternal viraemia postpartum, maternal HIV seroprevalence and ART coverage during ANC positively correlated with cases of MTCT, while higher proportions of women with fANC booking $<20$ weeks' gestation were associated with a decline in MTCT cases.

Conclusions. Findings suggest that maternal viraemia postpartum, geographical areas with a higher burden of maternal HIV, women initiating ART late in pregnancy and/or incident maternal HIV during pregnancy are significant population-level predictors of MTCT in the national prevention of MTCT programme. Scale-up of HIV prevention services is required to lower maternal HIV prevalence, while expanded access to HIV testing will fast-track ART initiation among WRLHIV. Increased VL monitoring is critical to improve VL suppression rates for elimination of MTCT.
\end{abstract}

S Afr Med J 2022;112(3):219-226. https://doi.org/10.7196/SAMJ.2022.v112i3.16041

South Africa (SA) has a well-established and mature prevention of mother-to-child transmission of HIV (PMTCT) programme, in existence for close to two decades. ${ }^{[1]}$ PMTCT services are available in $>95 \%$ of all public health facilities in the country, and HIV testing and antiretroviral therapy (ART) initiation among pregnant women were near universal by $2017 .^{[1,2]}$ In the same year, the proportion of first antenatal care (fANC) bookings occurring before 20 weeks' gestation had increased to $67 \%$ nationally, up from $54 \%$ in $2014 .{ }^{[3]}$ These data show that the national PMTCT programme had met targets for process indicators for validation for the elimination of mother-to-child transmission of HIV (eMTCT) in 2017, defined as $\geq 95 \%$ coverage for antenatal care, HIV testing of pregnant women and ART initiation among HIV-positive pregnant women. ${ }^{[4,5]}$ Since inception of the PMTCT programme in the early 2000s, the national early mother-to-child transmission (MTCT) transmission rate (4 6 weeks of age) declined significantly from 25 - 30\% in 2001 to $1.4 \%$ in 2016. ${ }^{[1]}$ By 2017, the national intrauterine (IU) rate ( $<7$ days of life) had decreased to $0.9 \%{ }^{[6]}$ These data demonstrate that SA is on a path towards eMTCT. However, the national PMTCT programme will need to overcome many challenges before it can achieve eMTCT.

The impact targets for eMTCT require reduction of MTCT rates to $<50$ cases per 100000 live births at cessation of breastfeeding. ${ }^{[4,5]} \mathrm{An}$ IU transmission rate of $0.9 \%$ equates to a case rate of $\sim 240$ per 100000 live births, excluding intra- and postpartum transmissions, in SA. The high case rate is attributable to a high maternal seroprevalence rate, which has plateaued at $30 \%$ since $2003 .{ }^{[7]}$ Moreover, maternal seroconversion during pregnancy and the breastfeeding period is common. ${ }^{[1]}$ Further reduction of MTCT rates to levels sufficient 
to meet eMTCT targets will require universal early diagnosis of HIV, ART initiation and viral suppression to $<50$ copies $/ \mathrm{mL}$ among pregnant women of reproductive age living with HIV (WRLHIV). ${ }^{[8]}$ This strategy should also be extended to non-pregnant WRLHIV to ensure virological suppression at the time of conception. Sustained maternal viral load $(\mathrm{VL})$ suppression $(\mathrm{VL}<50$ copies $/ \mathrm{mL}$ ) regardless of pregnancy status will fast-track eMTCT. However, VL monitoring among pregnant and breastfeeding WRLHIV has been suboptimal. ${ }^{[9,10]}$

Maternal VL information is required for infant risk stratification and determining the nature of infant prophylaxis, as well as maintaining maternal health. Calls have been made to prioritise VL monitoring among pregnant and postpartum WRLHIV in order to fast-track eMTCT in SA. ${ }^{[1,12]}$ Currently there is limited information on rates of $\mathrm{VL}$ suppression among pregnant and breastfeeding WRLHIV accessing care in the national PMTCT programme. Using data from 2014 to 2017, 30 - 35\% of pregnant WRLHIV delivering in the public sector in SA have been reported as having a VL $\geq 50$ copies/mL at time of delivery. ${ }^{[13,14]}$ Women who have unplanned pregnancies, adolescent girls and young women (AGYW) and migrant mothers have been identified as high-risk groups for unsuppressed VLs as a result of factors including newly acquired infection, late booking and diagnosis, and suboptimal adherence to ART. ${ }^{[15-17]}$ Geospatial differences in terms of HIV prevalence, ART coverage and viral suppression rates exist in SA. ${ }^{[18]}$ Some provinces have higher proportions of WRLHIV and AGYW living with HIV than others. ${ }^{[19]}$ However, at the time of writing (December 2020), there had been no assessment of the geospatial distribution of high maternal VLs during pregnancy and the postpartum period and the potential effect this has on MTCT rates in SA. Identifying areas with a high burden of maternal viraemia during pregnancy will guide stakeholders on where to focus attention and resources for eMTCT.

\section{Objectives}

Using a national laboratory dataset and other routine HIV-related data sources, we describe population-level factors associated with cases of MTCT (from birth to $<24$ months postpartum) at subdistrict level in SA.

\section{Methods \\ Study design}

The study was an ecological analysis of routine laboratory HIVrelated test data from a synthetic cohort of pregnant and nonpregnant WRLHIV in SA in 2016 and 2017.

\section{Creation of study cohort}

Data were drawn from a study evaluating the evolution of maternal VLs during pregnancy, delivery and postpartum in the public health sector in SA. The data source and creation of the study cohort have been described elsewhere. ${ }^{[20]}$ In brief, a cohort of WRLHIV (age 15 - 49 years) was identified from the National Health Laboratory Service's Corporate Data Warehouse (NHLS CDW) between 2016 and 2017 using HIV VL and CD4 count data. Because the NHLS CDW lacks a marker for pregnancy, a set of criteria based on syphilis screening and timing of HIV-related tests such as CD4 counts and VLs was applied to the routine data to differentiate pregnant from non-pregnant WRLHIV ${ }^{[20]}$ For pregnant WRLHIV, follow-up started at cohort entry (which was the fANC visit based on syphilis test date), through delivery to exit at the latest VL and up to 15 months post delivery. ${ }^{[20]}$ All VLs performed during follow-up were included. Eligible VLs were categorised into: (i) cohort entry VL: defined by the first VL performed during pregnancy, i.e. at fANC visit for ART- experienced pregnant WRLHIV or after 3 months of ART use for pregnant WRLHIV not on ART at fANC visit; (ii) delivery VL (5 7 months after the fANC visit); and (iii) cohort exit VL, defined by the most recent VL measurement within the 15-month postpartum period. Analysis was restricted to the first identified pregnancy, i.e. the first syphilis test date, and its associated follow-up VLs during the study period.

Since eMTCT requires viral suppression prior to pregnancy, we compared VLs of pregnant WRLHIV with those of non-pregnant WLRHIV at comparable time points. For non-pregnant WRLHIV, follow-up started at cohort entry on 1 January 2016 and exited at the latest VL conducted prior to 31 December 2018 or at the close of the dataset on 31 December 2018. In this group, the cohort entry VL constituted the first VL performed during the study period and the latest VL measurement during the study period constituted the cohort exit VL. In both groups, de-duplication of test records was performed by a validated, automated patient-linking algorithm that uses probabilistic matching of patient demographics (name, surname, date of birth and geographical location) in the NHLS CDW. ${ }^{[21]}$ Fig. 1 describes follow-up times stratified by pregnancy status.

Other routine indicators known to be associated with MTCT of HIV were extracted from the South African District Health Information System (DHIS) for the period 2016 - 2018. The DHIS has been described previously. ${ }^{[19]}$ Data on relevant indicators extracted at subdistrict level were 'Antenatal first visit $<20$ weeks rate' (ANC early booking) and 'Antenatal client start on ART rate' (ART coverage among women initiated prior to and during pregnancy). Lastly, district-level HIV prevalence rates among pregnant women were obtained from the latest national antenatal sentinel HIV and syphilis survey report (2017). ${ }^{[7]}$ Owing to unavailability of disaggregated data, district-level ANC HIV prevalence estimates were used for each subdistrict within that district. Table 1 defines population-level study variables (indicators) and their sources.

\section{Study measures}

Study outcome

The study outcome was number of MTCT cases, defined as the number of HIV polymerase chain reaction (PCR)-positive children aged $<24$ months between 2016 and 2018 in the public health sector. These test data were de-duplicated using the age at which the child first tested HIV PCR-positive. De-duplication of test data was achieved by automated probabilistic matching of patient demographics supplemented by manual linkage of patient demographic information (name, surname, date of birth and geographical location).

\section{Study exposures}

The main exposure variables for this study were the numbers of WRLHIV with a VL $\geq 50$ copies $/ \mathrm{mL}$ at specified time points: at cohort entry, at delivery (for pregnant WRLHIV), and at cohort exit. Other exposure variables included proportions of ANC early booking among pregnant women, maternal HIV prevalence, ART coverage rate during ANC among pregnant women, and number of WRLHIV aged $<25$ years, stratified by pregnancy status and geographical location. Patient-level data of pregnant and non-pregnant WRLHIV were aggregated and analysed at subdistrict level. The geographical location of study participants was assigned to the subdistrict where the entry VL was performed. Pregnant WRLHIV are a highly mobile population across healthcare facilities in SA. ${ }^{[22]}$ Mobility of the cohort across subdistricts was minimal based on $3.3 \%$ and $4.6 \%$ of the cohort moving across subdistricts between the first and subsequent VL test during follow-up for pregnant and non-pregnant WRLHIV, 


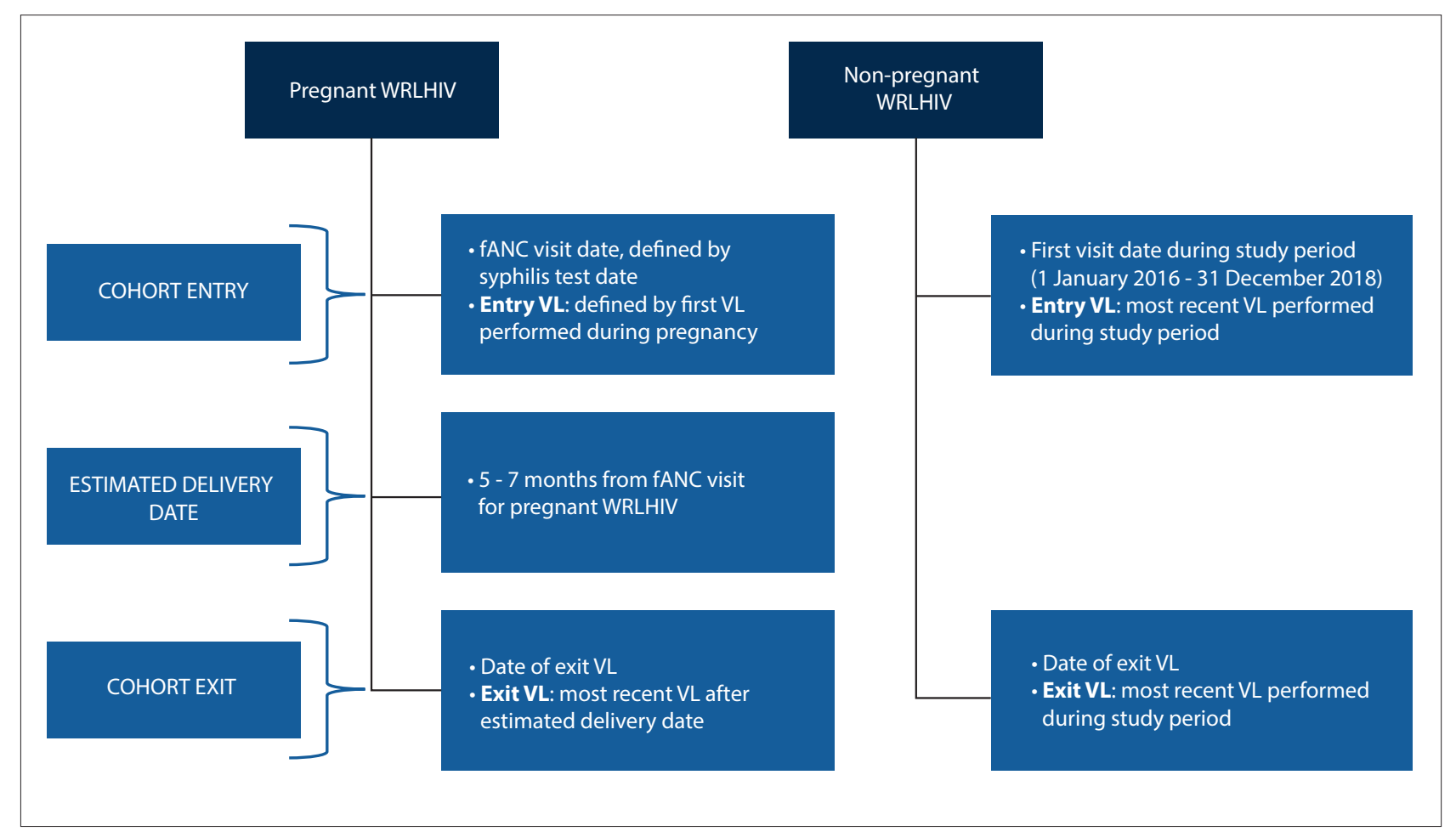

Fig. 1. Definition of follow-up time for pregnant and non-pregnant WRLHIV during the study period. (WRLHIV = women of reproductive age living with $H I V ; f A N C=$ first antenatal care; $V L=$ viral load.)

Table 1. Population-level variables, description and data sources

\begin{tabular}{|c|c|c|c|}
\hline Population-level variable & Description & Source & Data availability \\
\hline $\begin{array}{l}\text { Antenatal first visit before } 20 \\
\text { weeks rate }\end{array}$ & $\begin{array}{l}\text { Proportion of pregnant women with fANC booking } \\
\text { before } 20 \text { weeks' gestation from all fANC visits } \\
\text { recorded }\end{array}$ & DHIS & $\begin{array}{l}\text { District and subdistrict } \\
\text { level }\end{array}$ \\
\hline ANC ART coverage & $\begin{array}{l}\text { Proportion of pregnant women receiving ART during } \\
\text { pregnancy from all ANC attendees living with HIV }\end{array}$ & DHIS & $\begin{array}{l}\text { District and subdistrict } \\
\text { level }\end{array}$ \\
\hline ANC HIV prevalence rate & $\begin{array}{l}\text { Proportion of HIV-positive pregnant women from all } \\
\text { ANC attendees }\end{array}$ & $\begin{array}{l}\text { National Antenatal } \\
\text { Sentinel HIV and } \\
\text { Syphilis Survey Report }{ }^{[7]}\end{array}$ & District level \\
\hline Maternal age $<25$ years & $\begin{array}{l}\text { Number of WRLHIV aged }<25 \text { years at fANC visit/ } \\
\text { cohort entry stratified by pregnancy status }\end{array}$ & NHLS CDW & $\begin{array}{l}\text { District and subdistrict } \\
\text { level }\end{array}$ \\
\hline $\begin{array}{l}\text { Viraemia of pregnant and non- } \\
\text { pregnant WRLHIV }\end{array}$ & $\begin{array}{l}\text { Number of WRLHIV with VL } \geq 50 \text { copies } / \mathrm{mL} \text { at } \\
\text { specific time points during follow-up }\end{array}$ & NHLS CDW & $\begin{array}{l}\text { District and subdistrict } \\
\text { level }\end{array}$ \\
\hline MTCT cases & $\begin{array}{l}\text { Number of children testing HIV PCR-positive at } \\
<24 \text { months of age }\end{array}$ & NHLS CDW & $\begin{array}{l}\text { District and subdistrict } \\
\text { level }\end{array}$ \\
\hline
\end{tabular}

respectively. Analysis was restricted to participants with at least two VL measurements during follow-up.

\section{Data analysis}

Percentages (medians with interquartile ranges (IQRs)) were used to describe the population-level variables. ArcGIS version 10.8 (Esri, USA) was used to describe the geospatial distribution of maternal viraemia at delivery, expressed as percentage maternal VL $\geq 50$ or $\geq 1000$ copies $/ \mathrm{mL}$ by district. A negative binomial regression model was used to determine the association between cases of MTCT and the number of WRLHIV with unsuppressed VL at specific time points, i.e. at cohort entry, at delivery (for pregnant WRLHIV) or at cohort exit, controlling for subdistrict-level proportions of pregnant women with early ANC booking, ART coverage during ANC, ANC HIV prevalence, and number of women aged $<25$ years at fANC visit or cohort entry (study covariates). Variable selection was achieved by backward stepwise regression analysis using a statistical significance level of $20 \%$. Since younger maternal age is associated with vertical transmission of HIV, ${ }^{[23]}$ the number of WRLHIV aged $<25$ years at cohort entry was included into the model $a$ priori.

Two sensitivity analyses were performed, comparing the ability of different models in predicting an eMTCT target of 50 MTCT cases adjusting for study covariates at subdistrict level. The first analysis compared a negative binomial regression model using absolute numbers of maternal $\mathrm{VL}>50$ copies $/ \mathrm{mL}$ at specific time points adjusting for study covariates (model 1) v. a model using proportions 
of maternal VL $>50$ copies $/ \mathrm{mL}$ at specific time points adjusting for study covariates (model 2). The second analysis compared model 1 with a model using absolute numbers of maternal VL $>50$ copies $/ \mathrm{mL}$ at specific time points as a covariate unadjusted (model 3). Model comparison in either scenario relied on comparison of the area under the receiver operating characteristic (ROC) curves at the 5\% level of significance.

\section{Ethics considerations}

Ethical clearance was obtained from the Human Research Ethics Committee of the University of the Witwatersrand (ref. no. M180854). Access to data from the national early infant diagnosis programme was covered by ethical clearance issued to the National Institute for Communicable Diseases (ref. no. M160667).

\section{Results}

\section{Description of pregnant and non-pregnant WRLHIV} included in the study

A total of 3386507 WRLHIV from 262 subdistricts were identified from test records archived in the NHLS CDW, of whom 178319 (5.3\%) met criteria for pregnancy and 3208188 (94.7\%) did not. The median (IQR) age was 29.4 (24.6 - 33.7) years at fANC visit for pregnant WRLHIV and 34.0 (33.0 - 35.0) years at cohort entry for non-pregnant WRLHIV $(p<0.001)$ (Table 2). During the study period, the median proportion of women with early first booking during ANC $(<20$ weeks' gestation) was $68.2 \%(62.9-72.8)$ at subdistrict level. At the same time, the median ANC HIV prevalence rate per district was 31.5\% (23.4 35.7). ART coverage during ANC was almost universal, with a median coverage rate of $94.8 \%(89.7-97.8)$

\section{Unsuppressed VL among pregnant and non-pregnant WRLHIV}

Overall, the median (IQR) subdistrict-level proportion of WRLHIV with a VL $\geq 50$ copies/mL at cohort entry was $41.7 \%$ (27.8 - 57.8), with each woman contributing a median of $2.2(2-3)$ VLs during followup. When stratified by pregnancy status, the median subdistrict level proportion of pregnant WRLHIV with a VL $\geq 50$ copies $/ \mathrm{mL}$ at cohort entry was $42.9 \%(38.3-59.3)$ v. $35.0 \%(25.9-49.0)$ for non-pregnant WRLHIV at cohort entry $(p<0.001)$. By the time of delivery, the proportion of pregnant WRLHIV with an elevated VL had decreased, resulting in a median subdistrict-level proportion of pregnant WRLHIV with a VL $\geq 50$ copies $/ \mathrm{mL}$ of $38.2 \%$ (27.3 52.1). However, maternal $\mathrm{VL} \geq 50$ copies $/ \mathrm{mL}$ at cohort exit remained higher when compared with non-pregnant WRLHIV, with a median of $36.3 \%(25.0-48.4)$ v. $29.6 \%(21.0-42.6)$, respectively $(p<0.001)$ (Table 2). A total of 4535 children aged $<24$ months tested HIV PCRpositive, representing a median total case rate of 1372 (914 - 2 077) per 100000 live births at subdistrict level during the study period.

\section{Geospatial distribution of maternal viraemia at delivery by district}

At delivery, $36.9 \%$ and $14.3 \%$ of pregnant WRLHIV were viraemic at $\mathrm{VL} \geq 50$ and $\geq 1000$ copies $/ \mathrm{mL}$, respectively, at national level. At district level, proportions of pregnant WRLHIV with a VL $\geq 50$ copies/ $\mathrm{mL}$ at delivery varied greatly, ranging from $18 \%$ to $61 \%$. Proportions of maternal viraemia at delivery were highest in Limpopo Province, with at least $50 \%$ of WRLHIV having VL $\geq 50$ copies $/ \mathrm{mL}$ at delivery in all 5 districts (Fig. 2). A similar trend was observed in 3/5 districts in Gauteng Province (Ekurhuleni, Sedibeng and West Rand), 1/4 districts in Free State Province (Fezile Dabi) and 2/8 districts in Eastern Cape Province (Sarah Baartman and Nelson Mandela). Namakwa district in Northern Cape Province also had a high proportion of maternal $\mathrm{VL} \geq 50$ copies/mL at delivery, probably owing to very low numbers of pregnant WRLHIV. District-level proportions of maternal VL $\geq 1000$ copies $/ \mathrm{mL}$ at delivery followed a similar distribution. Districts with the highest proportions of women with a VL $<50$ copies $/ \mathrm{mL}$ at delivery were mostly located in KwaZulu-Natal Province (Fig. 2).

\section{Population-level factors associated with vertical transmission of HIV}

Viraemia during the postpartum period for pregnant WRLHIV, ANC HIV prevalence rate and ANC first booking <20 weeks' gestation rate were associated with the number of MTCT cases in the univariate analysis (Table 3). In the adjusted analyses, for every 100 WRLHIV with a VL $\geq 50$ copies/mL postpartum, the number of MTCT cases increased by 3 at subdistrict level (Fig. 3). Maternal HIV prevalence also positively correlated with number of children testing HIV PCR-positive, i.e. a $100 \%$ increase in subdistrict-level

Table 2. Population-level estimates related to mother-to-child transmission risk among WRLHIV in South Africa, 2016 - 2018 ( $N=262$ subdistricts)

\begin{tabular}{|c|c|c|c|c|}
\hline \multirow[b]{2}{*}{ Variable } & \multicolumn{4}{|c|}{ Subdistrict-level estimates, median (IQR) } \\
\hline & Overall & Pregnant WRLHIV & Non-pregnant WRLHIV & $p$-value \\
\hline Age at cohort entry (years) & $33.4(27.8-39.6)$ & $29.4(24.6-33.7)$ & $34.0(33.0-35.0)$ & $<0.001^{*}$ \\
\hline fANC visit $<20$ weeks' gestation rate, $\%$ & - & $68.2(62.9-72.8)$ & - & - \\
\hline ANC HIV prevalence, $\%$ & - & $31.5(23.4-35.7)$ & - & - \\
\hline ANC ART coverage, $\%$ & - & $94.8(89.7-97.8)$ & - & - \\
\hline MTCT case rate, /100 000 live births & - & $1372(914-2$ 077) & - & - \\
\hline \multicolumn{5}{|l|}{ HIV VL $\geq 50$ copies $/ \mathrm{mL}, \%$} \\
\hline At cohort entry & $41.7(27.8-57.8)$ & $42.9(38.3-59.3)$ & $35.0(25.9-49.0)$ & $<0.001^{\star}$ \\
\hline At delivery & - & $38.2(27.3-52.1)$ & - & - \\
\hline At cohort exit & $34.6(21.6-44.1)$ & $36.3(25.0-48.4)$ & $29.6(21.0-42.6)$ & $<0.001^{\star}$ \\
\hline \multicolumn{5}{|l|}{ HIV VL $\geq 1000$ copies/mL, \% } \\
\hline At cohort entry & $19.8(14.6-26.4)$ & $35.8(28.7-42.1)$ & $18.3(14.4-24.7)$ & $<0.001^{\star}$ \\
\hline At delivery & - & $15.6(12.5-21.6)$ & - & - \\
\hline At cohort exit & $13.3(10.1-17.5)$ & $15.4(11.3-20.0)$ & $13.8(10.1-19.2)$ & $<0.001^{\star}$ \\
\hline
\end{tabular}




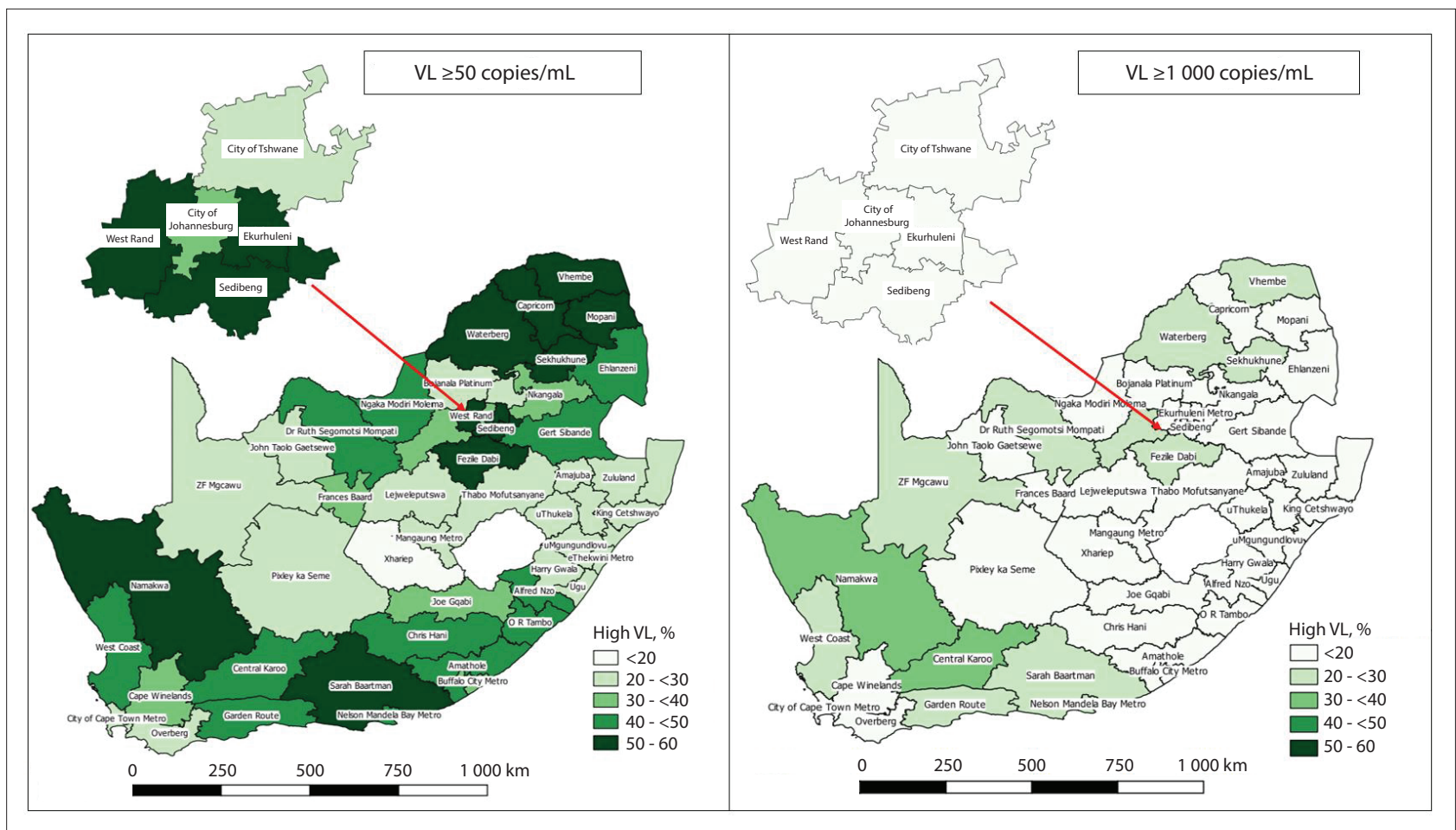

Fig. 2. Maternal viraemia at delivery by district in South Africa, 2016 - 2018. (VL = viral load.)

Table 3. Variable selection for multivariable model for subdistrict-level factors associated with vertical transmission of HIV from birth to $<24$ months of age in South Africa, 2016 - 2018

\begin{tabular}{|c|c|c|}
\hline \multirow[b]{2}{*}{ Variable } & \multicolumn{2}{|c|}{$\begin{array}{c}\text { Full model (backward elimination) } \\
\text { Selection cut-off: } p<0.20\end{array}$} \\
\hline & $p$-value & Selection \\
\hline ANC HIV prevalence rate & $0.067^{*}$ & Included \\
\hline Number of pregnant WRLHIV aged $<25$ years at cohort entry & 0.320 & Included a priori \\
\hline Number of pregnant WRLHIV with VL $\geq 50$ copies/mL at cohort entry & 0.372 & Excluded \\
\hline Number of pregnant WRLHIV with VL $\geq 50$ copies /mL at delivery & 0.677 & Excluded \\
\hline Number of pregnant WRLHIV with VL $\geq 50$ copies $/ \mathrm{mL}$ at cohort exit & $<0.001^{*}$ & Included \\
\hline Number of non-pregnant WRLHIV aged $<25$ years at cohort entry & $0.090^{*}$ & Included \\
\hline Number of non-pregnant WRLHIV with VL $\geq 50$ copies $/ \mathrm{mL}$ at cohort entry & 0.969 & Excluded \\
\hline Number of non-pregnant WRLHIV with VL $\geq 50$ copies $/ \mathrm{mL}$ at cohort exit & $<0.001^{*}$ & Included \\
\hline ART coverage among ANC clients rate & $0.187^{\star}$ & Included \\
\hline ANC first booking $<20$ weeks' gestation rate & $0.003^{*}$ & Included \\
\hline
\end{tabular}

maternal HIV prevalence increased the number of children testing HIV PCR-positive by 2 (Fig. 3). An inverse association between the proportion of women with first ANC booking $<20$ weeks' gestation and the number of cases of MTCT was observed. A $100 \%$ increase in the proportion of WRLHIV booking at a gestational age $<20$ weeks decreased the number of children testing HIV PCR-positive by 2 at subdistrict level (Fig. 3). An increase in ART coverage was also associated with an increase in cases of MTCT, suggesting increasing ART coverage among women presenting late in pregnancy and/or cases of incident maternal HIV during pregnancy. A separate model adjusting for maternal VL threshold $\geq 1000$ copies $/ \mathrm{mL}$ at the same time points during follow-up yielded similar results.

Results from the sensitivity analyses demonstrated that there was no difference in the accuracy of predicting the eMTCT target of
50 cases using the model including absolute numbers of maternal viraemia $v$. the model using proportions of maternal viraemia at specific time points as a covariate in the adjusted analyses (ROC $p=0.789$ ) (Fig. 4A). The model using absolute numbers of maternal VL at specific time points adjusting for study covariates was superior to the model using maternal VL at specific time points unadjusted. However, the difference was not statistically significant (ROC $p=0.166$ ) (Fig. 4B).

\section{Discussion}

We present findings from an analysis evaluating subdistrict-level determinants of MTCT in SA, at a time when the national PMTCT programme is working towards achieving eMTCT. Understanding factors driving MTCT across the country will assist in developing 


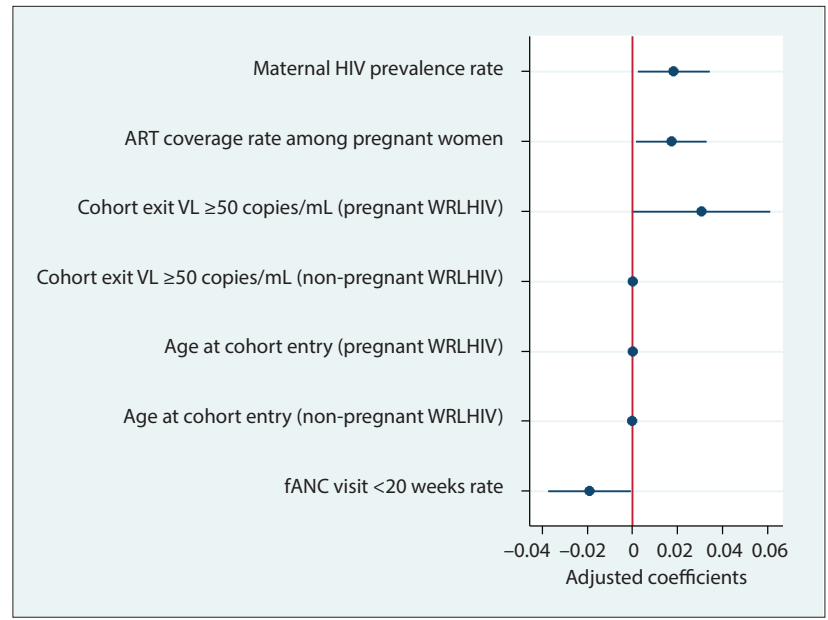

Fig. 3. Negative binomial regression model showing subdistrict-level factors associated with vertical transmission of HIV from birth to $<24$ months of age in South Africa, $2016-2018(\mathrm{~N}=204$ subdistricts, those with missing data excluded (listwise deletion)). $(A R T=$ antriretroviral therapy; $V L=$ viral load; $W R L H I V=$ women of reproductive age living with HIV; fANC = first antenatal care.)

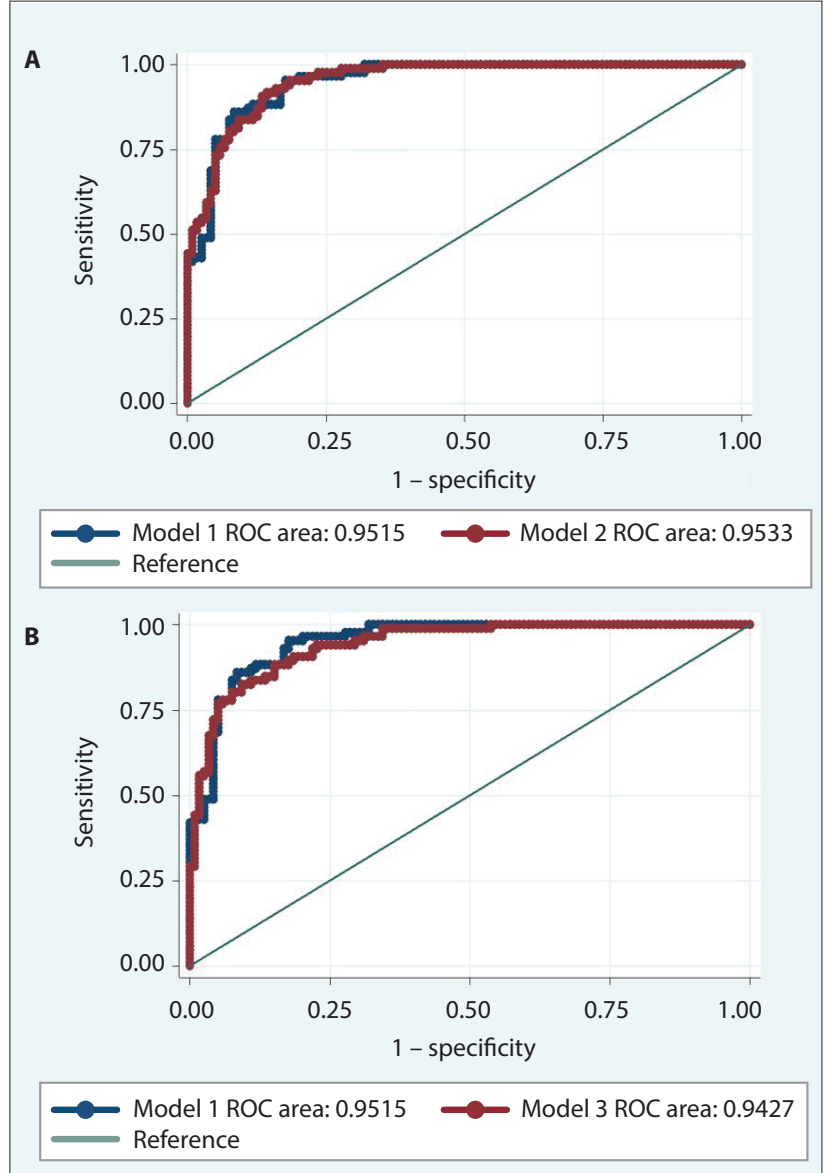

Fig. 4. Sensitivity analyses. ROC curves comparing: (A) model 1 (using absolute number of maternal $V L \geq 50$ copies $/ m L$ ) v. model 2 (\% maternal $V L \geq 50$ copies $(\mathrm{mL}$ ) at specific time points as a covariate adjusted for other study covariates; and (B) model $1 v$. model using absolute number of maternal VL at specific time points unadjusted (model 3$).(R O C=$ receiver operating curve; $V L=$ viral load.) targeted interventions to facilitate eMTCT. The study found that proportions of unsuppressed VLs were higher among pregnant WRLHIV compared with non-pregnant WRLHIV throughout follow-up. For example, median proportions of women with viraemia (VL $\geq 50$ copies $/ \mathrm{mL})$ were $43 \%$ v. $35 \%$ at cohort entry $(p<0.001)$ and $36 \%$ v. $30 \%$ at most recent VL measurements $(p<0.001)$ for pregnant and non-pregnant WRLHIV, respectively. Maternal viraemia during the postpartum period correlated positively with cases of vertical transmission, with every 100 viraemic postpartum WRLHIV increasing the number of MTCTs by 3 cases. Furthermore, higher rates of HIV prevalence among ANC clients were associated with a higher number of MTCT cases, whereby a $100 \%$ increase in maternal HIV prevalence resulted in an increase of MTCT cases by 2 at subdistrict level. The same trend was observed for ART coverage during ANC among pregnant women. A higher proportion of women booking early for ANC was associated with a decline in the number of HIV PCR-positive children. District-level differences in proportions of maternal viraemia ( $\mathrm{VL} \geq 50$ or $\geq 1000$ copies $/ \mathrm{mL}$ ) at delivery were noted across the country.

These findings are among few examining population-level determinants of vertical transmission of HIV in the national PMTCT programme in the era of near-universal rates of maternal ART coverage. Previous studies and surveys using data ranging from 2010 to 2013 reported a positive association between early MTCT rates and suboptimal PMTCT service coverage (provincial maternal ART coverage rate $<80 \%$ and insufficient healthcare personnel in facilities), ${ }^{[24]}$ and recommended early treatment of maternal HIV as a way of reducing vertical transmission. ${ }^{[24]}$ Almost a decade later, our data show that PMTCT coverage (ART coverage among ANC clients) was near universal at 95\%, and the proportion of ANC clients booking early for care was almost $70 \%$ during the study period nationally. These programmatic changes may have contributed to the further decline in early ( $4-8$ weeks postpartum) MTCT rates from $3.5 \%$ to $1.5 \%$ (7 days - $<10$ weeks postpartum) between 2010 and 2017. ${ }^{[1,25]}$ Early ART initiation during pregnancy is known to be protective against MTCT of HIV. ${ }^{[26,27]}$ However, in the present study, results of the adjusted regression model showed increased vertical transmission with increasing ART coverage among pregnant women. This finding is suggestive of increased ART coverage among women newly diagnosed during pregnancy due to seroconversion. Approximately 1 in 2 pregnant WRLHIV included in the study were ART naive at the fANC visit, ${ }^{[20]}$ and these figures were similar to reports from the DHIS during the study period. As a result, even though ART coverage during pregnancy was high, women initiating ART (particularly late in pregnancy) or seroconverting during pregnancy would not have had sufficient time to suppress their VL, hence transmitting HIV to their children. Moreover, high levels of viraemia have been documented from ART-experienced women at the fANC visit from a separate analysis using the same data as this study. ${ }^{[20]}$ ART coverage therefore does not equate to reduced MTCT, as it does not take into account VL suppression. Achieving eMTCT will require early diagnosis of HIV and early ART initiation for all pregnant WRLHIV to ensure sustained maternal VL suppression. This can be facilitated by early booking for ANC. Findings continue to show that early booking for ART, and by extension, early treatment for maternal HIV, is still relevant in the programme and is associated with a lower number of MTCT cases. This suggests that the programme needs to continue to raise awareness of the importance of early attendance for ANC and the need for repeat HIV testing among HIV-negative women throughout pregnancy and postpartum to facilitate early ART initiation to prevent MTCT. ${ }^{[26-28]}$ 
Findings showed that maternal viraemia during the postpartum period was associated with MTCT of HIV at subdistrict level. This finding concurs with reports from other studies that show higher rates of MTCT during the postnatal period in routine settings. ${ }^{[23,29,30]}$ These findings emphasise the need for continued engagement in PMTCT care and VL monitoring among postpartum WRLHIV to prevent MTCT. Disengagement from care among postpartum WRLHIV is common in SA and other high HIV burden countries and may lead to women becoming viraemic as a result of non-adherence to ART. ${ }^{[31-33]}$ In SA, this has important programmatic implications given the extended breastfeeding period of up to 24 months among WRLHIV on ART. ${ }^{[34]}$ In order to achieve eMTCT, the programme requires better systems for tracking WRLHIV throughout pregnancy and the postpartum period to enable retention in care and efficient VL monitoring to guide clinical management in this population. The study data did not show the effect of maternal viraemia at cohort entry and at delivery on cases of MTCT. This finding can be attributed to fewer VL measurements: the median (IQR) number of VLs was $2.2(2$ - 3) per woman during follow-up, so there were potentially insufficient data points to establish the longitudinal effect of viraemia on vertical transmission at subdistrict level, something that requires further study.

Ensuring that all WRLHIV are virally suppressed is probably one of the most practical ways of fast-tracking eMTCT in SA, given: (i) the magnitude of the programme; and (ii) inadequacies of other methods for fast-tracking eMTCT in these settings (breastfeeding cannot be avoided, and universal caesarean births are not feasible). ${ }^{[34,35]}$ The study data showed that community VLs were higher in pregnant WRLHIV than in non-pregnant WRLHIV throughout follow-up, suggesting that pregnancy was associated with lower rates of viral suppression. While there are no physiological reasons why pregnancy should correlate with lack of viral suppression, ${ }^{[36]}$ psychosocial factors related to pregnancy are known to affect maternal adherence to ART and subsequently lead to lack of VL suppression. ${ }^{[36]}$ A patientcentred approach towards managing pregnant WRLHIV is therefore recommended to improve viral suppression rates and prevent vertical transmission. It can be achieved by scaling up mentor-mother and community healthcare programmes to assist women in adhering to treatment from within their communities.

Lastly, varying proportions of maternal VL $\geq 50$ copies $/ \mathrm{mL}$ at delivery among districts were observed. High-burden areas for maternal viraemia at delivery were observed in Limpopo and Gauteng provinces, and in some parts of Eastern Cape and Mpumalanga. Pregnant WRLHIV accessing care in districts in KwaZulu-Natal and Western Cape provinces had better rates of VL suppression compared with the rest of the country. These district-level differences in maternal viraemia highlight high-burden areas for maternal viraemia in the country and the need for targeted responses. KwaZulu-Natal, despite having optimal maternal VL suppression rates overall, still had higher cases of MTCT on account of a high maternal seroprevalence rate. These data concur with results of the adjusted regression model whereby higher maternal seroprevalence rates correlated positively with number of MTCT cases. This finding further emphasises the need for targeted responses in order to achieve eMTCT. It is also worth noting that maternal VL suppression rates at the time of delivery may have improved subsequent to the study period. The 2019 guidelines now recommend a VL test at delivery for all pregnant WRLHIV in an attempt to prompt appropriate clinical management for mother-child pairs during the postpartum period, since the postnatal period is the new frontier of MTCT. ${ }^{[23,37]}$ In addition, the introduction of dolutegravir into standard first-line therapy may improve maternal VL suppression rates during pregnancy and the postpartum period. ${ }^{[37]}$

\section{Study limitations}

Interpretation of these findings requires acknowledgement of a number of limitations. Results for pregnant WRLHIV are based on a synthetic cohort that was created by applying a set of criteria to routine HIV test data owing to lack of a marker for pregnancy in the NHLS CDW. The generalisability of the study data therefore relies on the accuracy of algorithms used. It is anticipated that the study algorithm used to identify pregnant WRLHIV from the NHLS CDW was highly specific, resulting in a small number of women identified as pregnant during the study period. As a result, some pregnant WRLHIV were misclassified as being non-pregnant. However, results emanating from this study cohort are similar to results from other studies that enrolled known pregnant WRLHIV from routine settings. ${ }^{[13,38]}$

The lack of a unique patient identifier in the NHLS CDW may have resulted in poor linkage of VL test data, leading to under-estimation of VL suppression for both pregnant and non-pregnant WRLHIV. In addition, VL monitoring in the PMTCT programme was not widely implemented during the study period, so VL testing coverage was low. As a result, testing may have been biased towards women with unsuppressed VLs. Lastly, the ART status of pregnant women in our study was based on availability of monitoring VLs according to national guidelines. ${ }^{[20]}$ However, women may fall out of care and re-initiate treatment in pregnancy, resulting in some pregnant women being misclassified as ART naive in this study. However, the large sample size of the individual-level data potentially minimised this bias. With regard to MTCT cases, the reported national case rate may be over-estimated owing to decreased linkage in the NHLS CDW. On the other hand, the case rate may be an under-estimate because children diagnosed with HIV $<24$ months of age were tested using HIV PCR. The national PMTCT guidelines required HIV PCR testing in $<18$-month-olds and rapid HIV tests in those $\geq 18$ months of age during the study period. However, results of rapid HIV tests are not recorded in the NHLS CDW, so the study is likely to have missed MTCT cases in the 18 - 24 months age group.

On a population level, the analysis was based on 262 data points representing subdistricts in the country. A facility-level analysis would have provided a larger dataset, with greater geographical variability in the exposures and outcomes. However, some of the indicators analysed were not available at facility level, restricting analysis to subdistrict level. Furthermore, the ecological design of this study makes it liable to ecological fallacy, i.e. findings from a population-level analysis may not apply to a patient-level analysis. For example, subdistrict-level data did not show the effect of younger maternal age on MTCT, when patient-level analyses have shown that younger maternal age is a significant predictor of vertical transmission of HIV in these settings. ${ }^{[23,27]}$ Nonetheless, the data provide useful insight on population-level parameters that require addressing in order to fast-track eMTCT in SA.

\section{Conclusions}

Despite near-universal coverage of PMTCT services in SA, MTCT case rates at the end of the breastfeeding period are still in excess of eMTCT targets. Results from this ecological analysis suggest that maternal viraemia during the postpartum period, geographical areas with higher maternal seroprevalence rates, women initiating ART late in pregnancy and/or incident maternal HIV during pregnancy are significant population-level predictors of vertical transmission 
in the national PMTCT programme. Scale-up of HIV prevention such as family planning services is required to lower maternal HIV prevalence, while expanded access to HIV testing will fast-track ART initiation among WRLHIV. Retention in care and increased VL monitoring among pregnant and postpartum WRLHIV, with prompt clinical action for abnormal results, are critical to improve VL suppression rates and maternal health throughout the continuum of PMTCT care for eMTCT.

Declaration. The research for this study was done in partial fulfilment of the requirements for FM's PhD in Public Health at the University of the Witwatersrand.

Acknowledgements. The authors acknowledge the NHLS CDW for providing laboratory test data.

Author contributions. FM, TK, GGS and AHM conceived the study design and selected indicators for analysis. GGS and FM acquired the data. FM, AHM, TK and GGS analysed/interpreted the data. FM wrote the article. TK, AHM and GGS provided critical revision and final approval. Funding. The authors acknowledge financial support for the study from the ELMA Foundation.

Conflicts of interest. None.

1. Goga A, Sherman G, Chirinda W, et al. Eliminating mother-to-child transmission of HIV in South Africa, 2002 - 2016: Progress, challenges and the Last Mile Plan. In: Barron P, Padarath A. Twenty years of the South African Health Review: South African Health Review 20th edition. Durban: Health Systems Trust, 2017:137-146. https://hdl.handle.net/10520/EJC-c80ada3a4 (accessed 18 January 2022). 2. Bhardwaj S, Treger-Slavin L, Barron P, et al. Elimination of mother-to-child transmission of HIV in South Africa: Rapid scale-up using quality improvement. S Afr Med J 2014;104(3):239-243. https:// doi.org/10.7196/SAMJ.7605

3. Massyn N, Padarath A, Peer N, Day C. District Health Barometer 2016/2017. Durban: Health Systems Trust, 2016. https://www.hst.org.za/publications/Pages/District-Health-Barometer-201617.aspx Trust, 2016.

. World Health Organization. Global Guidance on Criteria and Processes for Validation: Elimination 4. World Health Organization. Global Guidance on Criteria and Processes for Validation: Elimination
of Mother-to-child Transmission (EMTCT) of HIV and Syphilis. 2014. https://www.who.int/ of Mother-to-child Transmission (EMTCT) of HIV and Syphilis. 2014. https:/,
reproductivehealth/publications/emtct-hiv-syphilis/en/ (accessed 22 November 2020).

reproductivehealth/publications/emtct-hiv-syphilis/en/ (accessed 22 November 2020).
5. World Health Organization. Global Guidance on Criteria and Processes for Validation: Elimination World Health Organization. Global Guidance on Criteria and Processes for Validation: Elimination
of Mother-to-child Transmission (EMTCT) of HIV and Syphilis. 2nd ed., 2017. https://www.who.int reproductivehealth/publications/emtct-hiv-syphilis/en/ (accessed 22 November 2020).

6. Goga A, Chirinda W, Ngandu NK, et al. Closing the gaps to eliminate mother-to-child transmission of HIV (MTCT) in South Africa: Understanding MTCT case rates, factors that hinder the monitorin and attainment of targets, and potential game changers. S Afr Med J 2018;108(3a):s17-s24. https://dol. org/10.7196/SAMJ.2017.v108i3b.12817

7. Woldesenbet SA, Kufa T, Lombard C, et al. The 2017 National Antenatal Sentinel HIV Survey, South Africa, National Department of Health. 2019. https://www.nicd.ac.za/wp-content/uploads/2019/07/ Antenatal_survey-report_24July19.pdf (accessed 18 July 2020).

8. Mandelbrot L, Tubiana R, le Chenadec et al. No perinatal HIV-1 transmission from women with effective antiretroviral therapy starting before conception. Clin Infect Dis 2015;61(11):1715-1725. effective antiretroviral therapy sta

9. Lesosky M, Glass T, Mukonda E, Hsiao N-E, Abrams EJ, Myer L. Optimal timing of viral load Lesosky M, Glass T, Mukonda E, Hsiao N-E, Abrams EJ, Myer L. Optimal timing of viral load
monitoring during pregnancy to predict viraemia at delivery in HIV-infected women initiating ART in
South Africa: A simulation study. J Int AIDS Soc 2017;20(7):e25000. https://doi.org/10.1002/jia2.25000 South Africa: A simulation study. J Int AIDS Soc $2017 ; 20(7): 225000$. https://doi.org/10.1002/jia2.25000
0. Abougi LL, Humphrey JM, Mpondy C, et al. Achieving UNAIDS 90-90-90 targets for pregnant and postpartum women in sub-Saharan Africa: Progress, gaps and research needs. J Virus Erad 2018;4(2):33-39. https://doi.org/10.1016/s2055-6640(20)30343-5

11. Myer L, Essajee S, Broyles LN, et al. Pregnant and breastfeeding women: A priority population for HIV viral load monitoring. PLoS ONE 2017;14(8):e1002375. https://doi.org/10.1371/journal. pmed. 1002375

12. Onoya D, Nattey C, Jinga N, Mongwenyana C, Sherman G. Time of HIV diagnosis, CD4 count and viral load at antenatal care start and delivery in South Africa. PLoS ONE 2020;15(2):e0229111. https:// doi.org/10.1371/journal.pone.0229111

13. Woldensenbet S, Kufa T, Barron P, et al. Viral suppression and factors associated with failure to achieve viral suppression among pregnant women in South Africa. AIDS 2020;34(4):589-597. https://doi. viral suppression among pregnant

14. Myer L, Phillips TK, McIntyre JA, et al. HIV viraemia and mother-to-child transmission risk after Myer L, Phillips TK, McIntyre JA, et al. HIV viraemia and mother-to-child transmission risk after
antiretroviral therapy initiation in pregnancy in Cape Town, South Africa. HIV Med 2017;18(2):80-88. antiretroviral therapy initiation in
https://doi.org/10.1111/hiv.12397
15. Fatti G, Shaikh N, Eley B, Jackson D, Grimwood A. Adolescent and young pregnant women at increased risk of mother-to-child transmission of HIV and poorer maternal and infant health outcomes: A cohort study at public facilities in the Nelson Mandela Bay metropolitan district, Eastern Cape, South Africa. study at public facilities in the Nelson Mandela Bay metropolitan district,
S Afr Med J 2014;104(12):874-880. https://doi.org/10.7196/SAMI.8207

16. Ramraj T, Jackson D, Dinh T-H, et al. Adolescent access to care and risk of early mother-to-child HIV transmission. J Adolesc Health 2018;62(4):434-443. https://doi.org/10.1016/j.jadohealth.2017.10.007

17. Kim H-Y, Dobra A, Tanser F. Migration and first-year maternal mortality among HIV-positive postpartum women: A population-based longitudinal study in rural South Africa. PLoS Med 2020;17(3):e1003085. https://doi.org/10.1371/journal.pmed.1003085

18. Johnson LF, Dorrington RE, Moolla H. HIV epidemic drivers in South Africa: A model-based evaluation of factors accounting for-inter-provincial differences in HIV prevalence and incidence trends. South Afr J HIV Med 2017;18(1):a695. https://doi.org/10.4102/sajhivmed.v1811.695

19. Moyo F, Mazanderani AH, Kufa T, Sherman G. The geographic distribution of priority population groups for the elimination of mother-to-child transmission of HIV in South Africa. PLoS ONE 15(4):e0231228. https://doi.org/10.1371/journal.pone.0231228

20. Moyo F, Mazanderani AH, Murray T, Sherman GG, Kufa T. Achieving maternal viral load suppression for Moyo F, Mazanderani AH, Murray T, Sherman GG, Kufa T. Achieving maternal viral load suppression for
elimination of mother-to-child transmission of HIV in South Africa. AIDS 2021;35(2):307-316. https:// doi.org/10.1097/qad.0000000000002733

21. Bassett IV, Huang M, Cloete $C$, et al. Assessing the completeness and accuracy of South African National Laboratory CD4 and viral load data: A cross-sectional study. BMJ Open 2018;8(8):e021506. https://doi. org/10.1136/bmjopen-2018-021506

22. Clouse K, Pettifor A, Shearer K, et al. Loss to follow-up before and after delivery among women testing HIV positive during pregnancy in Johannesburg, South Africa. Trop Med Int Health 2013;18(4):451-460. https://doi.org/10.1111/tmi.12072

23. Chetty T, Newell M-L, Thorne C, Coutsoudis A. Viraemia before, during and after pregnancy in HIVinfected women on antiretroviral therapy in rural KwaZulu-Natal, South Africa, 2010 - 2015. Trop Med Int Health 2018;23(1):79-91. https://doi.org/10.1111/tmi.13001

24. Woldesenbet SA, Jackson D, Lombard CJ, et al. Structural level differences in the mother-to-child HIV transmission rate in South Africa: A multilevel assessment of individual-, health facility-, and provinciallevel predictors of infant HIV transmission. J Acquir Immune Defic Syndr 2017;74(5):523-530. https:// doi.org/10.1097/qai.0000000000001289

25. Goga A, Jackson D, Lombard C, et al. Highest risk of mother-to-child transmission of HIV or death in the first 6 months postpartum: Results from 18 month follow-up of an HIV-exposed cohort, South Africa. In: first 6 months postpartum: Results from 18 month follow-up of an HIV-exposed cohort, South Africa. In: AIDS Soc 2016;19(6 Suppl 5):27-28. https://doi.org/10.7448/IAS.19.6.21264

26. Technau KG, Kalk E, Coovadia A, et al. Timing of maternal HIV testing and uptake of prevention of mother-to-child transmission interventions among women and their infected infants in Johannesburg, South Africa. J Acquir Immune Defic Syndr 2014;65(5):e170-e178. https://doi.org/10.1097/ qai.0000000000000068

27. Kendall C, Claessens L, Dorward J, Mfeka G, Gate K. Reasons for failure of prevention of mother-to-child HIV transmission in a rural South African district hospital. South Afr J HIV Med 2015;16(1):365-368. https://doi.org/10.4102/sajhivmed.v16i1.365

28. Johnson LF, Stinson K, Newell ML, et al. The contribution of maternal HIV seroconversion during late pregnancy and breastfeeding to mother-to-child transmission of HIV. J Acquir Immune Defic Syndr 2012;59(4):417-425. https://doi.org/10.1097/qai.0b013e3182432f27

29. Gill MM, Hoffman HJ, Bobrow EA, et al. Detectable viral load in late pregnancy among women in the Rwanda Option B+ PMTCT Program: Enrollment results from the Kabeho Study. PLoS ONE 2016;11(12):e0168671. https://doi.org/10.1371/journal pone. 0168671

30. Myer L, Dunning L, Lesosky M, et al. Frequency of viremic episodes in HIV-infected women initiating antiretroviral therapy during pregnancy: A cohort study. Clin Infect Dis 2017;64(4):422-427. https://doi. $\mathrm{org} / 10.1093 / \mathrm{cid} /$ ciw792

31. Adeniyi OV, Ajayi AI. Level and determinants of postpartum adherence to antiretroviral therapy in the Eastern Cape, South Africa. PLoS ONE 2020;15(2):e0229592. https://doi.org/10.1371/journal. pone.0229592

32. Phillips T, Thebus E, Bekker L-G, Mcyntire J, Abrams EJ, Myer L. Disengagement of HIV-positive pregnant and postpartum women from antiretroviral therapy services: A cohort study. J Int AIDS Soc 2014;17(1):19242. https://doi.org/10.7448/ias.17.1.19242

33. Nachega JB, Uthman OA, Anderson J, et al. Adherence to antiretroviral therapy during and after pregnancy in low-income, middle-income and high-income countries: A systematic review and metaanalysis. AIDS 2012;26(16):2039-2052. https://doi.org/10.1097/qad.0b013e328359590f

34. Le Roux SM, Abrams EJ, Donald KA, et al. Infectious morbidity of breastfed, HIV-exposed uninfected infants under conditions of universal antiretroviral therapy in South Africa: A prospective cohort study. Lancet Child Adolesc Health 2020;4(3):220-231. https://doi.org/10.1016/S2352-4642(19)30375-X

35. Egbe TO, Tchente CN, Mangala Nkwele G-F, Nyemb JE, Barla ME, Belley-Priso E. Cesarean delivery technique among HIV positive women with sub-optimal antenatal care uptake at the Douala General Hospital, Cameroon: Case series report. BMC Res Notes 2017;10:332. https://doi.org/10.1186/s13104Hospital, Can
$017-2639-0$

36. Westreich D, Cole SR, Nagar S, Maskew M, van der Horst C, Sanne I. Pregnancy and virologic response to antiretroviral therapy in South Africa. PLoS ONE 2011;6(8):e22778. https://doi.org/10.1371/journal. pone. 0022778

37. National Department of Health, South Africa. Guideline for the Prevention of Mother to Child Transmission of Communicable Infections (HIV, Hepatitis, Listeriosis, Malaria, Syphilis and TB). https:// www.nicd.ac.za/wp-content/uploads/2019/11/Guidelines-for-the-Prevention-of-Transmission-ofCommunicable-Diseases-from-mother-to-child_28-October.pdf (accessed 1 February 2022).

38. Moyo F, Haeri Mazanderani A, Murray T, et al. Characterizing viral load burden among HIV-infected women around the time of delivery: Findings from four tertiary obstetric units in Gauteng, South Africa. I Acquir Immune Defic Syndr 2020;83(4):390-396. https://doi.org/10.1097/qai.0000000000002267

Accepted 8 December 2021 